\title{
Efeito da ordem dos exercícios no treinamento de força sobre a pressão arterial pós esforço em idosas hipertensas
}

http://dx.doi.org/10.11606/1807-5509201900020323

\author{
Diogo CARDOZO ${ }^{* * *}$ \\ Ana Paula VASCONCELOS ${ }^{* *}$ \\ Tiago FIGUEIREDO ${ }^{* * * * *}$ \\ Roberto SIMÃO* \\ *Escola de Educação \\ Física e Desportos, \\ Universidade Federal \\ do Rio de Janeiro, Rio \\ de Janeiro, RJ, Brasil. \\ ${ }^{* *}$ Faculdade Aberta a \\ Melhor Idade, Instituto \\ Metodista Granbery, \\ Juiz de Fora, MG \\ Brasil. \\ ***Universidade \\ Estácio de Sá, Rio de \\ Janeiro, RJ, Brasil.
}

\section{Resumo}

0 objetivo deste estudo foi analisar o efeito de diferentes ordens de exercícios sobre a pressão arterial (PA) após sessões de treinamento de força (TF). Quinze idosas hipertensas inexperientes em TF foram divididas em dois grupos. Grupo de membros superiores (G1) e grupo membros inferiores (G2). 0 G1 realizou três séries de 15 repetições submáximas na sequência de exercícios: Supino Reto (SR), Remada Fechada (RF), Tríceps no Puxador (TP) e Rosca Bíceps (RB). A segunda sequência do $G 1$ foi inversa (RB, TP, RF, SR). 0 G2 realizou quatro séries de 15 repetições submáximas na sequência de exercícios: Leg Press (LP), Cadeira Extensora (CE) e Flexão Plantar (FP). A segunda sequência do G2 foi inversa FP, CE, LP. A PA foi mensurada em repouso e após as sessões de exercícios durante 60 minutos. Para análise dos dados, utilizou-se uma ANOVA de dois caminhos com medidas repetidas e post hoc de Tukey para verificar as diferenças na PA antes e após as sessões de treinamento. Ambos os grupos apresentaram reduções significativas na PA sistólica pós-exercício com a sequência de exercícios do grande para o pequeno grupo muscular $\mathrm{G} 1$ (20, 30 e $40 \mathrm{~min})$ e $\mathrm{G} 2$ (30, 40, 50 e $60 \mathrm{~min})$. Quando a sequência foi inversa, foram observadas diferenças significativas em alguns momentos $\mathrm{G} 1$ (30 e $40 \mathrm{~min}$ ) e $\mathrm{G} 2$ (40 e $50 \mathrm{~min}$ ). Não foram encontradas diferenças significativas entre os grupos e também para a PA diastólica em qualquer sequência. Os resultados indicam que quando a sequência de exercícios é iniciada do grande para o pequeno grupo muscular, há uma tendência para maior duração do efeito hipotensivo.

Palavras-Chave: Exercícios Resistidos; Idosos; Pressão Arterial; Hipotensão.

\section{Introdução}

A hipertensão arterial (HA) afeta mais de $25 \%$ da população mundial sendo fator de risco primário para doença arterial coronariana, infarto agudo do miocárdio, doenças arteriais periféricas e falência renal $^{1}$. O desenvolvimento e progressão da HA são associados a diversos fatores como inatividade física, genética, envelhecimento, sobrepeso e obesidade, intolerância a glicose, resistência à insulina e baixa reatividade vascular ${ }^{1}$.

Dados da literatura demonstram que aproximadamente $30 \%$ da população brasileira desenvolve HA, e dentre estas, 50\% são idosas ${ }^{2,3}$. Desta forma, atitudes intervencionistas farmacológicas e não farmacológicas são necessárias para a prevenção e controle da pressão arterial (PA). Dentre as ações não farmacológicas, a prática de exercícios físicos regulares como tratamento tem se mostrado eficiente a longo prazo para o controle da $\mathrm{PA}^{1,4}$. O Exercícios físico atua com o efeito hipotensivo que é caracterizado pela redução da PA abaixo dos valores mensurados na condição de repouso, o que de certa forma contribui para prevençấo e controle da HA a longo prazo ${ }^{1,4}$. 
Dentre os diversos tipos de exercícios físicos, o treinamento de força (TF) tem sido pesquisado nos últimos anos com o objetivo de reduzir a $\mathrm{PA}^{5,6}$. Em posicionamento oficial o Colégio Americano de Medicina do Esporte ${ }^{1}$ recomenda que uma sessão de TF seja aplicada em complemento a uma sessão de treinamento aeróbico para esta finalidade. Após este posicionamento, alguns trabalhos foram realizados, demonstrando o efeito do TF realizado de forma isolada na resposta hipotensiva pós-exercício ${ }^{5-8}$. Contudo, mesmo sendo uma crescente esta linha de pesquisa, ainda há muito que investigar no que se refere ao controle das variáveis metodológicas do TF e sua influência sobre as respostas cardiovasculares após sessões de TF. Por exemplo, a maioria dos estudos encontrados na literatura concentra-se nas variáveis de intensidade e volume de treinamento ${ }^{5-10}$. Sendo assim, outras variáveis como a ordem dos exercícios não recebem atenção por parte da literatura cientifica, uma vez que a prescrição do TF envolve muitas variáveis ${ }^{11}$. Após ampla revisão de literatura, apenas três estudos foram encontrados com o objetivo de verificar a influência da ordem dos exercícios sobre o efeito hipotensivo após uma sessão de TF, e seus resultados são divergentes ${ }^{12-14}$. Por exemplo, no estudo de JANNIG et al. ${ }^{12}$, foi observado que a ordem dos exercícios influenciou na duração do efeito hipotensivo, com as respostas hipotensivas sendo significativas com o protocolo alternado por seguimento em idosos de ambos os sexos. Já no estudo de Figueiredo et al. ${ }^{13}$, foi investigado o efeito em homens jovens normotensos, ao qual, não foram verificadas respostas hipotensivas, porém, através $\mathrm{da}$ análise da variabilidade da frequência cardíaca, foi observado maior estresse cardíaco no período do pós-exercício quando os exercícios iniciaram dos membros superiores e progrediram em direção aos membros inferiores.

BENTES et al. ${ }^{14}$, investigaram o efeito da associação de diferentes ordens e intensidades de treinamento no efeito hipotensivo em mulheres treinadas. Os autores verificaram que tanto a ordem quanto a intensidade exercem influência na duração do efeito hipotensivo. Entretanto, mesmo existindo estudos sobre o tema, a literatura ainda é incipiente sobre diferentes ordenaçōes de exercícios no TF e sua influência nas respostas cardiovasculares. Outro ponto a ser considerado é sobre a amostra investigada, pois dos estudos existentes as investigações foram baseadas em diferentes níveis de condicionamento físico (destreinados e treinados) e o estado da saúde (normotensos e hipertensos) $)^{5,12,15,16}$. Desta forma, a proposta do presente estudo tem importante aplicabilidade prática na tentativa de verificar o efeito de diferentes ordenaçóes de exercícios sobre a resposta da PA após sessóes de TF em um público específico de mulheres idosas hipertensas e inexperientes em TF.

Diante do exposto acima, o objetivo deste estudo foi o de analisar o efeito de diferentes ordenaçóes de exercícios para membros superiores e inferiores sobre a resposta da PA após sessóes de TF realizado por mulheres idosas hipertensas.

\section{Método}

\section{Amostra}

Participaram deste estudo 15 mulheres idosas hipertensas pertencentes a um projeto orientado de atividade física para a terceira idade. Todas as voluntárias do estudo eram inexperientes em $\mathrm{TF}$ e foram previamente diagnosticadas com HA controlada, ao qual faziam uso de pelo menos um medicamento. As voluntarias foram distribuídas de forma intencional nos grupos de treinamento (G1 ou G2) de acordo com o uso da classe de medicamentos na tentativa de equilibrar os grupos com as diferentes classes medicamentosas (TABELA 1).

Todas as voluntárias foram avaliadas e liberadas para a inclusão do presente estudo por um médico cardiologista. As características gerais da amostra estão descritas na TABELA 2.

Os critérios de exclusão do presente estudo foram: a) as participantes não poderiam apresentar qualquer comprometimento muscular ou articular que influenciasse na mecânica do movimento durante as sessóes de TF; b) as participantes não poderiam apresentar outra doença cardiovascular ou metabólica, que pudesse modificar a resposta cardiovascular durante e/ou após as sessóes de TF; c) as participantes náo poderiam ter experiência prévia em TF; d) as participantes não poderiam realizar outra forma de exercício físico durante o período da coleta de dados, para que não ocorresse variação sobre a PA; e) as participantes selecionadas deveriam estar no estágio de hipertensão 1 . 
As participantes foram informadas sobre todos os procedimentos, possíveis riscos e benefícios e assinaram um termo de consentimento livre e esclarecido de acordo com a resoluçáo 196/96 do Conselho Nacional de Saúde. Este estudo foi aprovado pelo comitê de ética em pesquisa da Faculdade de Ciências Médicas da Suprema, Juiz de Fora, MG, sob o parecer 325.150. Antes do inicio do experimento, todas as voluntárias foram orientadas a não ingerir cafeína, bebidas alcoólicas ou fumar, e a manterem os seus hábitos alimentares durantes todo o período do estudo. Todos estes procedimentos foram adotados para não influenciar na PA durante a coleta de dados.

\section{Procedimentos}

\section{Teste de 15 repetições máximas (15RM)}

Durante a primeira visita ao laboratório a massa corporal e a estatura foram medidas através de uma balança e um estadiômetro (Filizola, Brasil). O teste de $15 \mathrm{RM}$ iniciou com o aquecimento com $50 \%$ da carga prevista para $15 \mathrm{RM}$. Posteriormente a carga foi aumentada para a primeira tentativa de 15RM. Se a tentativa fosse bem sucedida, cinco minutos de intervalo eram dados até a próxima tentativa. Cada voluntária teve até cinco tentativas em cada exercício, com intervalo de 10 minutos entre os exercícios para determinar as cargas de 15RM. Esse procedimento foi repetido até a carga máxima para 15RM fosse executada. Os testes foram repetidos 72 horas depois para estabelecer a reprodutibilidade da medida ${ }^{17}$.

Com o objetivo de reduzir a margem de erro no teste de 15RM foram adotadas as seguintes estratégias: a) Todas as voluntárias foram instruídas antes do teste, de modo que estivessem cientes de toda a rotina que envolvia a coleta de dados; b) A avaliada foi instruída sobre a técnica de execução dos exercícios através da familiarização com os aparelhos e com a execuçáo dos movimentos dos exercícios; c) $\mathrm{O}$ avaliador esteve atento quanto à posição adotada pela praticante, no momento da medida, pois pequenas variaçóes do posicionamento das articulaçôes envolvidas no movimento poderiam acionar outros músculos, levando a interpretaçóes errôneas dos escores obtidos; d) Os testes foram realizados no mesmo horário do dia; e) Todas as avaliadas permaneceram sem treinar os grupos musculares utilizados por um período mínimo de $72 \mathrm{~h}$ antes da realização das avaliaçóes ${ }^{17}$.
Inicialmente as voluntárias do estudo foram divididas em dois grupos: (1) grupo de treinamento de membros superiores (G1) e (2) grupo de treinamento de membros inferiores (G2). Posteriormente, submetidas a duas semanas de familiarização nos exercícios propostos totalizando quatro sessóes. O protocolo de adaptaçáo consistia de realizarem duas séries de 15 repetiçóes com carga leve de acordo com a percepção de esforço de cada participante baseado na escala de percepção de Omne-RES ${ }^{18}$. O G1 realizou os testes de 15RM na seguinte ordem de exercícios: supino reto (SR), remada fechada (RF), tríceps no puxador (TP) e rosca bíceps (RB). O G2 realizou os testes de 15RM na sequência de exercícios: leg press horizontal (LP), cadeira extensora (CE) e flexão plantar (FP).

\section{Sessóes de exercícios}

\section{Desenho experimental}

Após a realização das sessões de familiarização e dos testes de carga, as voluntárias foram submetidas a duas sessôes com delineamento alternado de TF. O G1 realizou o treinamento com diferentes ordens para membros superiores e o G2 realizou o treinamento com diferentes ordens para membros inferiores. Na primeira sequência do G1 os exercícios para os grandes grupamentos foram realizados antes dos pequenos (MS G-P) os exercícios foram organizados na seguinte ordem: SR, RF, TP e RB. Na segunda sequência do G1, a situação foi inversa, iniciando a sequência com os pequenos grupamentos musculares e progredindo para os maiores grupos musculares (MS P-G), RB, TP, RF e SR. Para a primeira sequência do G2 de membros inferiores (MI G-P) a ordem dos exercícios foi: LP, CE e FP. Já para a segunda sequência do G2 (MI P-G) a ordem foi inversa FP, CE e LP (FIGURA 1).

Para o G1 foram aplicadas três séries de 15 repetições com $70 \%$ da carga de $15 \mathrm{RM}$ e intervalo de dois minutos entre as séries e exercícios (3 séries com 4 exercícios). Para o G2 foram realizadas quatro séries de 15 repetiçóes com $70 \%$ da carga de $15 \mathrm{RM}$ ( 4 séries com 3 exercícios) e intervalo entre as séries e exercícios de dois minutos. Este procedimento foi adotado com o objetivo de equilibrar o volume total de treinamento entre os grupos (número de séries $\mathrm{x}$ número de repetições $\mathrm{x}$ número de exercícios). Os dois fatores que diferenciaram os grupos foram à ordem dos exercícios e a localização (membros inferiores $\times$ membros superiores). 
G-P: Sequência de exercícios do grande para os pequenos grupamentos musculares; P-G: Sequência de exercícios do pequeno para os grandes grupamentos musculares. $1^{\circ} \mathrm{dia}$

Anamnese, procedimentos experimentais, TCLE

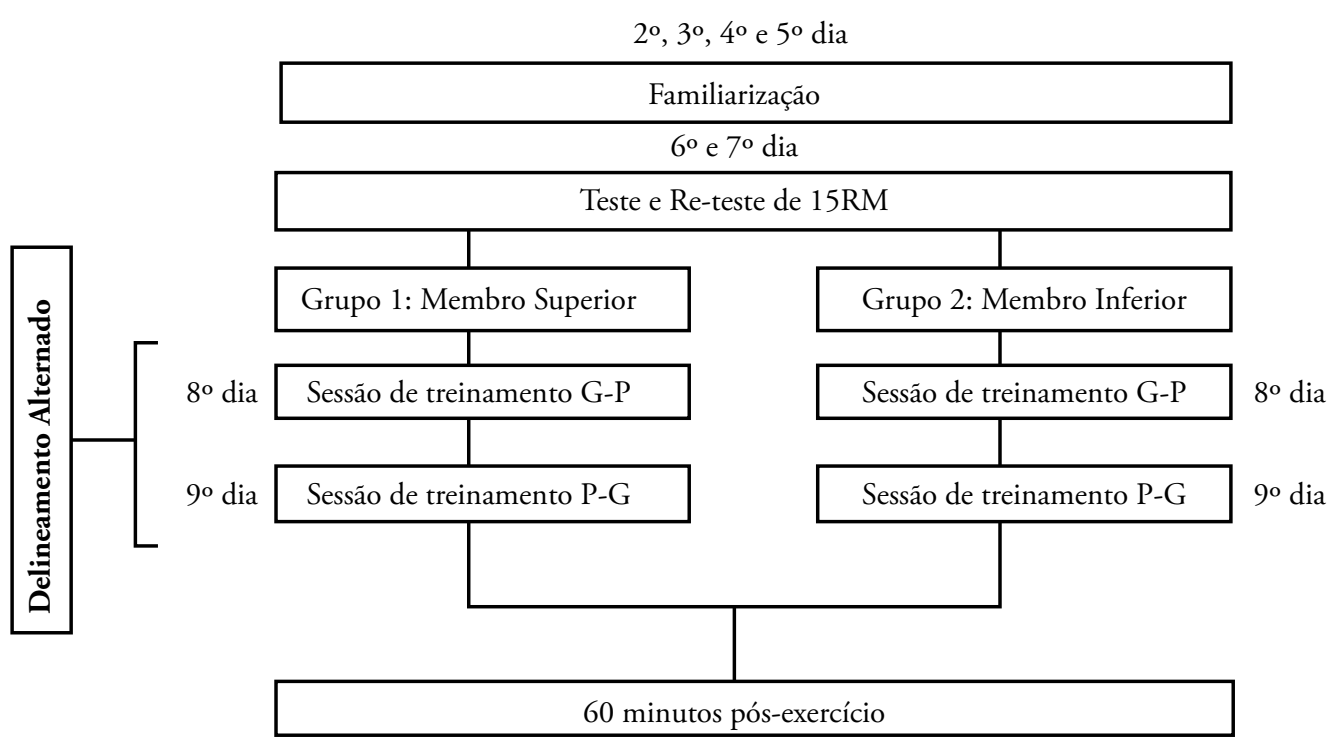

FIGURA 1 - Desenho experimental do estudo.

\section{Medidas da Pressão Arterial (PA)}

Antes do início de cada sessão de TF, as voluntárias permaneceram durante 10 minutos em ambiente calmo e tranquilo para a medida da PA de repouso. Após as sessóes de TF, a PA foi medida em ciclos de 10 minutos durante 60 minutos, resultando em seis medidas após a sessão de treinamento. Para isso, as medidas da PA foram feitas, indiretamente, pelo método auscultatório utilizando o aparelho de coluna de mercúrio (UNITEC, BRASIL). Todos os procedimentos foram realizados de acordo com as recomendaçóes das VI Diretrizes Brasileiras de Hipertensão ${ }^{2}$, que preconiza de não estar com a bexiga cheia, não praticar exercícios físicos pelo menos 60 minutos antes das medidas de repouso, não ingerir bebidas alcoólicas, café, alimentos e fumar pelo menos nos 30 minutos anteriores. $\mathrm{Na}$ tentativa de reduzir as probabilidades de erros, um único avaliador experiente foi padronizado para realizar todas as medidas da PA. Foram considerados como valor de PA sistólica (PAS) e PA diastólica (PAD) o primeiro e o quinto sons de Korotkoff respectivamente. Durante o monitoramento das medidas cardiovasculares, todas as voluntárias permaneceram na posição sentada ${ }^{4}$.

\section{Análise estatística}

Para verificar a normalidade dos dados da amostra foi aplicado o teste de Shapiro Wilk e homedasticidade (Critério de Bartlett). Para as variáveis que tiveram distribuição normal, uma ANOVA de um caminho foi aplicada para comparar os seguintes parâmetros: Teste e reteste de 15RM, valores de repouso da PAS e PAD. Posteriormente, os valores de repouso e após a sequência de treinamento foram comparados dentro de uma sessão e entre as diferentes sessões através da ANOVA de dois caminhos com medidas repetidas seguida do teste post hoc de Tukey quando necessário, para verificar as possíveis diferenças na PA antes e após as sessóes de treinamento. Adicionalmente, para determinar a magnitude dos resultados, tamanhos de efeito (diferença da média entre o pré-teste e pós-teste dividido pelo desvio-padrão do pré-teste) foram calculados para a PAS e PAD em todas as sequências de exercícios. A escala proposta por RHEA ${ }^{19}$ foi usada para determinar a magnitude dos resultados. Foi adotado um nível de significância de $\mathrm{p}<0,05$. Os procedimentos foram realizados através do software estatístico SPSS 20.0. 


\section{Resultados}

A TABELA 2 demonstra os dados gerais da amostra em ambos os grupos.

A TABELA 3 apresenta os valores da PAS nos dois grupos com as diferentes sequências de exercícios. Não foram observadas diferenças entre os valores de repouso dentro dos grupos $(\mathrm{G} 1 \mathrm{p}=0,380$ e G2 $\mathrm{p}=0,478)$ e entre os grupos $(\mathrm{p}=0,114$ e $\mathrm{F}=2,871$ para o dia de repouso das sessóes iniciadas dos grandes grupos musculares em direção aos pequenos grupamentos) e ( $\mathrm{p}=0,389$ e $\mathrm{F}=0,795$ para o dia de repouso das sessóes iniciadas dos pequenos grupamentos musculares em direção aos grandes grupos musculares). Ambos os grupos apresentaram reduçôes significativas na PA sistólica no período do pós-exercício com a sequência de exercícios do grande para o pequeno grupo muscular: o G1 aos 20 , 30 e 40 minutos e o G2 aos 30, 40, 50 e 60 minutos.
Quando a sequência de exercícios foi invertida, foram observadas diferenças significativas em alguns momentos, por exemplo, no G1 aos 30 e 40 minutos e o G2 observado diferença aos 40 e 50 minutos. Não foram encontradas diferenças significativas entre os grupos de treinamento.

A TABELA 4 demonstra os valores da PAD em ambos os grupos com diferentes ordenaçóes. Não foram observadas diferenças significativas em nenhum momento do período pós-exercício em relação aos valores de repouso e nem entre os grupos de treinamento com as diferentes sequências de exercícios.

A TABELA 5 apresenta a análise do tamanho do efeito dos valores da PAS e PAD respectivamente. De acordo com o modelo proposto por RHEA ${ }^{19}$, os valores se apresentam com classificação de trivial e pequeno.

TABELA 1 - Classes de medicamentos anti-hipertensivos

\begin{tabular}{lcc}
\hline $\begin{array}{l}\text { Medicamentos } \\
\text { anti-hipertensivos }\end{array}$ & $\begin{array}{c}\text { Grupo 1 } \\
\text { Número de sujeitos }\end{array}$ & $\begin{array}{c}\text { Grupo 2 } \\
\text { Número de sujeitos }\end{array}$ \\
\hline Betabloqueador & 2 & 2 \\
Inibidor da ECA & 3 & 2 \\
Diurético & 3 & 2 \\
Inibidor de canais de cálcio & - & 1 \\
\hline
\end{tabular}

TABELA 2 - Características gerais da amostra (Média \pm desvio-padrão)

\begin{tabular}{lcc}
\hline Variáveis & Grupo 1 & Grupo 2 \\
\hline N. Amostral & 8 & 7 \\
Idade (anos) & $69,9 \pm 5,6$ & $69,7 \pm 5,9$ \\
Massa corporal $(\mathrm{kg})$ & $67,2 \pm 13,4$ & $62,1 \pm 9,9$ \\
Estatura $(\mathrm{cm})$ & $156,5 \pm 7,1$ & $153,0 \pm 4,4$ \\
\hline
\end{tabular}

TABELA 3 - Comportamento da pressão arterial sistólica (Média \pm desvio-padrão)

\begin{tabular}{lcccc}
\hline & \multicolumn{2}{c}{ Membros superiores } & \multicolumn{2}{c}{ Membros Inferiores } \\
\cline { 2 - 5 } & G-P & P-G & G-P & P-G \\
\hline Repouso & $129,8 \pm 15,2$ & $130,8 \pm 13,4$ & $141,1 \pm 9,7$ & $136,6 \pm 11,6$ \\
10 minutos & $126,0 \pm 12,6$ & $127,8 \pm 16$ & $136,0 \pm 13,9$ & $134,3 \pm 10,8$ \\
20 minutos & $124,0 \pm 12,6^{*}$ & $124,5 \pm 15$ & $134,3 \pm 15,0$ & $132,9 \pm 11,8$ \\
30 minutos & $123,0 \pm 11,2^{*}$ & $122,8 \pm 11,2^{*}$ & $129,4 \pm 16,8^{*}$ & $130,9 \pm 12,3$ \\
40 minutos & $125,0 \pm 11,4^{*}$ & $123,3 \pm 12,7^{*}$ & $132,0 \pm 17,0^{*}$ & $129,7 \pm 13,1^{*}$ \\
50 minutos & $127,3 \pm 13,2$ & $125,0 \pm 17,1$ & $130,3 \pm 18,3^{*}$ & $129,7 \pm 12,4^{*}$ \\
60 minutos & $128,0 \pm 14,2$ & $127,0 \pm 15,6$ & $130,6 \pm 18,5^{*}$ & $130,6 \pm 12,4$ \\
\hline
\end{tabular}

G-P: sequência de exercícios do grande para 0 pequeno grupamento muscular; P-G: sequência de exercícios do pequeno para 0 grande grupamento muscular.

* Indica diferença significativa em relação ao repouso $\mathrm{p} \leq 0,05$. 
G-P: sequência de exercícios do grande para o pequeno grupamento muscular; P-G: sequência de exercícios do pequeno para 0 grande grupamento muscular.

TABELA 4 - Comportamento da pressão arterial diastólica (Média \pm desvio-padrão)

\begin{tabular}{lcccc}
\hline & \multicolumn{2}{c}{ Membros superiores } & \multicolumn{2}{c}{ Membros inferiores } \\
\hline Gepouso & G-P & P-G & G-P & P-G \\
10 minutos & $79,8 \pm 7,8$ & $78,0 \pm 9,4$ & $80,3 \pm 11,6$ & $80,6 \pm 10,6$ \\
20 minutos & $79,8 \pm 8,2$ & $76,5 \pm 8,2$ & $79,7 \pm 12,4$ & $80,6 \pm 10,3$ \\
30 minutos & $78,5 \pm 8,5$ & $78,0 \pm 11,2$ & $79,4 \pm 13,5$ & $79,7 \pm 11,7$ \\
40 minutos & $78,0 \pm 8,5$ & $76,0 \pm 9,1$ & $78,6 \pm 13,3$ & $78,3 \pm 13,0$ \\
50 minutos & $78,8 \pm 8,1$ & $77,3 \pm 9,4$ & $79,7 \pm 13,4$ & $77,4 \pm 14,1$ \\
60 minutos & $78,5 \pm 7,9$ & $76,5 \pm 9,4$ & $79,4 \pm 13,5$ & $77,7 \pm 13,7$ \\
& $78,8 \pm 7,9$ & $77,5 \pm 8,9$ & $79,1 \pm 13,2$ & $79,1 \pm 13,7$ \\
\hline
\end{tabular}

TABELA 5 - Tamanho do efeito da pressão arterial após as sessões de treinamento

MS: membro superior; Ml: membro inferior; PAS: pressão arterial sistólica;

PAD: pressão arterial diastólica;

Seq. G-P: sequência de exercícios do grande para 0 pequeno grupamento muscular; Seq. P-G: sequência de exercícios do pequeno para 0 grande grupamento muscular.

\begin{tabular}{|c|c|c|c|c|c|c|c|c|}
\hline & MS & & $10 \mathrm{~min}$. & $20 \mathrm{~min}$. & $30 \mathrm{~min}$. & $40 \mathrm{~min}$. & $50 \mathrm{~min}$. & $60 \mathrm{~min}$. \\
\hline \multirow[t]{10}{*}{ PAS } & Seq. & Magnitude & $-0,25$ & $-0,38$ & $-0,44$ & $-0,31$ & $-0,16$ & $-0,11$ \\
\hline & G-P & Classificação & Trivial & Trivial & Trivial & Trivial & Trivial & Trivial \\
\hline & Seq. & Magnitude & $-0,22$ & $-0,47$ & $-0,59$ & $-0,55$ & $-0,43$ & $-0,28$ \\
\hline & $\mathrm{P}-\mathrm{G}$ & Classificação & Trivial & Trivial & Peq. & Peq. & Trivial & Trivial \\
\hline & MI & & 10 min. & 20 min. & $30 \mathrm{~min}$. & $40 \mathrm{~min}$. & $50 \mathrm{~min}$. & 60 min. \\
\hline & Seq. & Magnitude & $-0,51$ & $-0,70$ & $-1,20$ & $-0,93$ & $-1,11$ & $-1,08$ \\
\hline & G-P & Classificação & Peq. & Peq. & Peq. & Peq. & Peq. & Peq. \\
\hline & Seq. & Magnitude & $-0,19$ & $-0,31$ & $-0,49$ & $-0,59$ & $-0,59$ & $-0,51$ \\
\hline & $\mathrm{P}-\mathrm{G}$ & Classificação & Trivial & Trivial & Trivial & Peq. & Peq. & Peq. \\
\hline & MS & & $10 \mathrm{~min}$. & $20 \mathrm{~min}$. & $30 \mathrm{~min}$. & $40 \mathrm{~min}$. & $50 \mathrm{~min}$. & $60 \mathrm{~min}$. \\
\hline \multirow[t]{9}{*}{ PAD } & Seq. & Magnitude & 0,00 & $-0,16$ & $-0,23$ & $-0,12$ & $-0,16$ & $-0,12$ \\
\hline & G-P & Classificação & Trivial & Trivial & Trivial & Trivial & Trivial & Trivial \\
\hline & Seq. & Magnitude & $-0,15$ & 0,00 & $-0,21$ & $-0,07$ & $-0,15$ & $-0,05$ \\
\hline & P-G & Classificação & Trivial & Trivial & Trivial & Trivial & Trivial & Trivial \\
\hline & MI & & $10 \mathrm{~min}$. & $20 \mathrm{~min}$. & 30 min. & $40 \mathrm{~min}$. & $50 \mathrm{~min}$. & $60 \mathrm{~min}$. \\
\hline & Seq. & Magnitude & $-0,05$ & $-0,07$ & $-0,14$ & $-0,05$ & $-0,07$ & $-0,1$ \\
\hline & G-P & Classificação & Trivial & Trivial & Trivial & Trivial & Trivial & Trivial \\
\hline & Seq. & Magnitude & 0,00 & $-0,08$ & $-0,21$ & $-0,3$ & $-0,27$ & $-0,14$ \\
\hline & P-G & Classificação & Trivial & Trivial & Trivial & Trivial & Trivial & Trivial \\
\hline
\end{tabular}

\section{Discussão}

O objetivo deste estudo foi analisar o efeito de diferentes ordenaçóes de exercícios para membros superiores e inferiores sobre a PA após TF realizado por mulheres idosas hipertensas. Para o conhecimento dos autores, este foi o primeiro estudo a comparar os efeitos de diferentes ordenaçóes de exercícios, utilizando duas diferentes sequências de TF para membros superiores e duas sequências para membros inferiores. Os principais achados do presente estudo apontam que: a) todas as sessóes de TF proporcionaram reduções dos níveis de PA pós-exercício em mulheres idosas hipertensas; b) a ordem dos exercícios não influenciou na magnitude do efeito hipotensivo pós-exercício, tanto no G1 quanto para o G2.

Nossos resultados demonstraram que uma única sessão de TF promove reduções significativas da PA em mulheres idosas hipertensas. O TF gerou efeito hipotensivo da PAS pós-exercício em todas as sessôes, contudo, ao fazer a análise pelo tamanho 
do efeito, foi observado que quando os exercícios iniciaram dos grandes grupos em direção aos pequenos grupamentos musculares, observaram-se diferentes tempos de duração do efeito hipotensivo quando comparados aos valores pré-exercício. Estas respostas foram observadas até os 30 minutos na ordem MS G-P (em três pontos de medidas, 20, 30 e 40min), e por 20 minutos na ordem MS P-G do G1 (em dois pontos de medidas 30 e 40min). Para o G2 foi observado na ordem MI G-P manutenção do efeito hipotensivo por 40 minutos (em quatro pontos de medidas, 30, 40, 50 e $60 \mathrm{~min}$ ) e na ordem MI P-G até 20 minutos (em dois pontos de medidas 40 e $50 \mathrm{~min}$ ).

Vários estudos já demonstraram que a ordem dos exercícios exercem influencia no desempenho durante uma sessão de $\mathrm{TF}^{21-23}$. Desta forma, exercícios que são realizados no início das sessões de treinamento apresentam melhor desempenho de repetiçóes do que quando são inseridos no final das sessóes de treinamento, ou seja, é indicado iniciar pelo grupamento muscular que seja prioridade ${ }^{23,24}$. Entretanto, baseado no tamanho do efeito, quando o objetivo for para a redução dos valores de PA, nossos resultados sugerem que iniciar os exercícios dos grandes grupamentos musculares e progredir para os pequenos grupamentos musculares, pode proporcionar maior duração do efeito hipotensivo da PAS, uma vez que quando a sessão de TF foi iniciada com os pequenos grupamentos musculares foi observado menor duração do efeito hipotensivo. Fato este, que deve ser levado em conta na prescrição de exercícios para indivíduos hipertensos.

Com relação da influência da ordem dos exercícios nas respostas da PA, foram encontrados apenas três estudos $^{12,13,14}$, sendo os resultados controversos. No estudo de JANNIG et al. ${ }^{12}$, a amostra foi composta por indivíduos idosos hipertensos de ambos os sexos que foram submetidos a três sessões de TF realizadas em diferentes ordenações de exercícios. No primeiro dia, foram realizados inicialmente os exercícios de membros inferiores e depois os de membros superiores. No segundo dia, a sequência foi realizada em ordem inversa, e no terceiro dia, os exercícios foram realizados em um sistema alternado por segmento. Somente o programa alternado por segmento proporcionou reduçáo significativa da PAS em todos os momentos investigados e da PAD em alguns momentos em relaçáo aos valores de repouso.

Já, no estudo de Figueiredo et al. ${ }^{13}$, foram avaliados indivíduos homens jovens normotensos que foram submetidos a duas diferentes sequências de TF: a sequência 1, iniciou com os exercícios de membros superiores e progrediu para os de membros inferiores e a sequência 2 com a ordem inversa. Não foram verificadas respostas hipotensivas, porém, foi observado maior estresse cardíaco através da medida da variabilidade da frequência cardíaca no período pós-exercício quando os exercícios iniciaram dos membros superiores em direção aos membros inferiores.

Bentes et al. ${ }^{14}$, avaliaram o efeito de diferentes ordenaçóes de exercícios e intensidades de treinamento sobre a resposta hipotensiva pós-exercício. Para isso, foram recrutadas 13 mulheres jovens experientes em TF que foram submetidas a quatro diferentes sessóes experimentais. A sequência A (SEQ-A) alternou exercícios de membros superiores e inferiores e a sequência B (SEQ-B) progredia os exercícios dos membros superiores em direção aos inferiores. Todas foram submetidas também a duas diferentes intensidades de treinamento com $60 \%$ e $80 \%$ de $1 \mathrm{RM}$. Desta forma as sessōes consistiam de (SEQ-A60\%, SEQ-A80\%, SEQ-B60\% e SEQ-B80\%). A PA foi medida em repouso, imediatamente após as sessóes de treinamento e no período do pós-exercício exatamente aos 15, 30, 45 e 60 minutos. Todas as voluntárias foram submetidas a três séries máximas com dois minutos de intervalo. Os autores observaram diminuiçóes significativas da PA após os diferentes protocolos de TF. Entretanto, para a duração a SEQ-A (60\% e 80 de $1 \mathrm{RM}$ ) foi mais efetiva no efeito hipotensivo, enquanto a SEQ-B somente com a intensidade com $80 \%$ de $1 \mathrm{RM}$. Desta forma, parece que intensidades maiores proporcionam maior duração do efeito hipotensivo e a sequência de exercícios que alterna membros superiores e inferiores é mais eficaz do que a progressão de exercícios de membros superiores em direção aos inferiores.

Uma das possíveis diferenças do presente estudo e dos resultados entre os estudos supracitados pode estar relacionada a fatores como idade, composiçáo da amostra, estado de saúde e de treinamento podem ter contribuído para estas distintas respostas. Por exemplo, no estudo de JANNig et al. ${ }^{12}$ foi investigado indivíduos idosos inexperientes em TF de ambos os sexos. Enquanto, nos estudos de Figueiredo et al. ${ }^{13}$ e Bentes et al. ${ }^{14}$ a amostra foi composta por indivíduos jovens experientes em TF. No presente estudo foi analisado um público especifico composto de mulheres com HA e os resultados apresentados podem servir para prescrição do TF para o grupo em questão.

Com relaçáo ao tamanho do grupamento muscular, os resultados do presente estudo demonstraram 
que não ocorreram diferenças entre os grupos de treinamento, porém o $\mathrm{G} 2$ apresentou uma tendência para maior duração do efeito hipotensivo quando comparada com o G1. Desta forma, o grupo de membros inferiores com a sequência de exercícios do grande em direção ao pequeno grupamento muscular proporcionou 10 minutos a mais de duraçáo do efeito hipotensivo do que o grupo de membros superiores com a mesma sequência.

Nossos resultados estão de acordo com outros estudos como os de Lizardo e Simóes ${ }^{25}$ e Polito e FARINATTi $^{8}$, que observaram que exercícios de membros inferiores proporcionam maior duração do efeito hipotensivo quando comparados a exercícios para membros superiores. Desta forma, a escolha do tamanho do grupamento muscular recrutado no inicio da sessão de treinamento pode afetar os valores da PA pós-exercício ${ }^{26}$. Algumas pesquisas demonstraram que exercícios que solicitam menores massas musculares proporcionam menores respostas pressóricas durante a execução, devido à pequena rede vascular que estará ocluída ${ }^{27,28}$. Contudo, após o término do exercício uma pequena quantidade de vasos sanguíneos estará dilatada. Por outro lado, exercícios que exijam maiores redes vasculares proporcionam maiores respostas pressóricas e prolongado efeito hipotensivo, devido as maiores quantidades de vasos em dilatação no pós-exercício ${ }^{20,26}$. Outras respostas fisiológicas também são verificadas na hipotensão tais como, redução da resistência vascular periférica, redução da descarga do nervo simpático e redução do débito cardíaco ${ }^{29}$. Algumas pesquisas tem verificado grande influência na redução do débito cardíaco por queda do volume plasmático tanto em indivíduos normotensos, quanto em hipertensos na redução dos níveis de PA pós-exercício ${ }^{9,30}$.

A PAS respondeu de forma eficaz a hipotensão pós-exercício enquanto que os valores da PAD não foram modificados em qualquer ponto de medida. Com resultados similares Fisher ${ }^{31}$, verificou que a sessão de TF em formato de circuito, foi capaz de induzir a resposta hipotensiva da PAS durante o período de recuperação em mulheres de meia-idade normotensas e hipertensas, entretanto sem diferenças significativas para a PAD.

A análise do tamanho do efeito indica valores redutores de pequena magnitude tanto para PAS, quanto para a PAD. Entretanto, mesmo que de pequena magnitude, os resultados apresentados tem importante aplicação clínica. É, bem consolidado na literatura que decréscimos de aproximadamente 3 $\mathrm{mmHg}$ na pressão arterial podem reduzir os riscos de acidente vascular cerebral em até $12 \%$, e de adquirir doença arterial coronariana em até $9 \%{ }^{1}$.

Uma das limitações do presente estudo está associada ao método utilizado para aferir a PA. O método invasivo é considerado como padrão-ouro para medida da PA, contudo, sua utilização pode gerar alguns riscos sendo inviável na prática ${ }^{32}$. Desta forma, mesmo o método auscultatório apresentando limitaçóes quando aplicado com rigor torna-se uma importante ferramenta de auxílio na determinação dos níveis pressóricos, uma vez que apresenta também boa correlação com outros métodos mais sofisticados de medida da $\mathrm{PA}^{33}$. É importante mencionar que o presente estudo constou de voluntárias que faziam uso de diferentes classes de medicamentos. É sabido que os agentes farmacológicos influenciam reduzindo o impacto da PA durante e após sessóes de exercício físico ${ }^{34,35}$. Contudo, pela metodologia do presente estudo foi tomado o cuidado de distribuir por igual às voluntárias com as diferentes classes de medicamentos nos diferentes grupos de treinamento na tentativa de equilibrar os mesmos. Outro ponto importante é que não foi realizada uma sessão controle para verificar as diferenças do dia de prática de exercício físico e o dia sem atividade para verificar o efeito do treinamento. Entretanto, é possível afirmar que uma sequência de treinamento proporciona maior duração do que a outra. A interpretação dos resultados também deve ser levada em conta, pois, o presente estudo foi realizado apenas com mulheres idosas hipertensas, portanto, nossos resultados são restritos ao grupo estudado.

De acordo com os resultados do presente estudo o TF aplicado com diferentes ordens é eficaz em proporcionar efeito hipotensivo em mulheres idosas hipertensas. A ordem dos exercícios náo influenciou na magnitude do efeito hipotensivo, contudo, foi observado que quando os exercícios iniciam com os grandes grupamentos musculares e progridem em direção aos pequenos grupamentos musculares, tanto para os membros superiores, quanto para os membros inferiores foi observada uma tendência para maior duração do efeito hipotensivo, mas esses resultados foram baseados no tamanho do efeito. Nesse sentido, parece que a ordem dos exercícios pode de alguma forma influenciar na duração, mas não na magnitude do efeito hipotensivo. Sugerimos que pesquisas adicionais sejam realizadas com outros grupamentos musculares e associação com outras variáveis do TF na tentativa de assegurar um adequado protocolo de treinamento com o objetivo de reduzir a PA de mulheres idosas hipertensas. 


\section{Abstract}

\section{Effect of order of exercise in strength in blood pressure post-exercise in elderly hypertensive}

The objective of this study was to analyze the effect of different exercise orders on blood pressure (BP) after strength training sessions (TF). Fifteen inexperienced elderly hypertensive were divided into two groups: Group upper (G1) and Group lower (G2). The G1 performed three sets of 15 repetitions at submaximal in exercise sequence: bench press (BP), low row (LR), triceps extension (TE) and biceps curl (BC). The second sequence of the $G 1$ was inverse ( $B C, T E, L R, B P)$. The $G 2$ held four 15 repetitions series in submaximal exercise sequence: leg press (LP), leg extension (LE) and plantar flexion (PF). The second sequence of $\mathrm{G} 2$ was reverse FP, CE, LP. BP was measured at rest and after exercise sessions for 60 minutes. For data analysis, we used an ANOVA two-way repeated measures and post hoc Tukey to verify differences in PA before and after the training sessions. Both groups showed significant reductions in post-exercise systolic BP with big sequence of exercises for small muscle group $\mathrm{G} 1(20,30$ and $40 \mathrm{~min})$ and $\mathrm{G} 2(30,40,50$ and $60 \mathrm{~min})$. When the sequence was reversed, significant differences were observed in some time G1 (30 to $40 \mathrm{~min}$ ) and G2 (40 to $50 \mathrm{~min}$ ). There were no significant differences between groups and for diastolic BP in any sequence. The results indicate that when the exercise sequence is initiated from large muscle groups, there is a tendency for increased duration of hypotensive effect.

KeYwoRds: Hypertension; Resistance Exercises; Elderly; Blood Pressure; Hypotension.

\section{Referências}

1. Pescatello LS, Franklin BA, Fagard R, et al. American College of Sports Medicine position stand. Exercise and hypertension. Med Sci Sports Exerc. 2004;36(3):533-53.

2. Sociedade Brasileira de Hipertensão, Sociedade Brasileira de Cardiologia, Sociedade Brasileira de Nefrologia. VI Diretrizes brasileiras de hipertensão. Arq Bras Cardiol. 2010;95(1 supl 1):1-51.

3. Picon RV, Fuchs FD, Moreira LB, Riegel G, Fuchs SC. Trends in prevalence of hypertension in Brazil: a systematic review with meta-analysis. PLoS One. 2012;7(10):e48255.

4. Braith RW, Stewart KJ. Resistance exercise training: its role in the prevention of cardiovascular disease. Circulation. 2006;113(22):2642-50.

5. Figueiredo $T$, Rhea MR, Peterson $M$, et al. Influence of number of sets on blood pressure and heart rate variability after a strength training session. J Strength Cond Res. 2015;29(6):1556-63.

6. Simão R, Fleck SJ, Polito M, Monteiro W, Farinatti PVT. Effects of Resistance training intensity, volume, and session format on post-exercise hypotension response. J Strength Cond Res. 2005;19(4):853-58.

7. Salles BF, Maior AS, Polito M, et al. Influence of rest interval lengths on hypotensive response after strength training sessions performed by older men. J Strength Cond Res. 2010;24(10):3049-54.

8. Polito MD, Farinatti PT. The effects of muscle mass and number of sets during resistance exercise on postexercise hypotension. J Strength Cond Res. 2009;23(8):2351-7.

9. Rezk CC, Marrache RC, Tinucci T, Mion D Jr, Forjaz CLM. Post-resistance exercise hypotension, hemodynamic, and heart rate variability: influence of exercise intensity. Eur J Appl Physiol. 2006;98(1):105-12.

10. Figueiredo T, Willardson JM, Miranda $\mathrm{H}$, et al. Influence of load intensity on post exercise hypotension and heart rate variability following a strength training session. J Strength Cond Res. 2015;29(10):2941-8.

11. American College of Sports Medicine. Progression models in resistance training for healthy adults. Med Sci Sports Exerc. 2009; 41(3):687-708.

12. Jannig PR, Cardoso AC, Fleischman E, Coelho CW, Carvalho T. Influencia da ordem de execução de exercícios resistidos na hipotensão pós-exercício em idosos hipertensos. Rev Bras Med Esporte. 2009;15(5):338-41.

13. Figueiredo T, Menezes P, Kattenbraker MS, et al. Influence of exercise order on blood pressure and heart rate variability after a strength training session. J Sports Med Phys Fitness. 2013;53(suppl 1):12-7. 
14. Bentes CM, Costa PB, Neto GR, et al. Hypotensive effects and performance responses between different resistance training intensities and exercise orders in apparently health women. Clin Physiol Funct Imaging. 2015;35(3):185-90. Epub 2014 Apr 1.

15. Scher LML, Ferriolli E, Moriguti JC, Scher R, Lima NKC. The effect of different volumes of acute resistance exercise on elderly individuals with treated hypertension. J Strength Cond Res. 2011;25(4):1016-23.

16. Melo CM, Alencar Filho AC, Tinucci T, Mion D Jr, Forjaz CLM. Post exercise hypotension induced by low-intensity resistance exercise in hypertensive women receiving captopril. Blood Press Monit. 2006;11(4):183-9.

17. Simão R, Farinatti PTV, Polito MD, Maior AS, Fleck SJ. Influence of exercise order on the number of repetitions performed and perceived exertion during resistance exercises. J Strength Cond Res. 2005;19(1):152-6.

18. Gearhart RF Jr, Lagally KM, Riechman SE, Andrews RD, Robertson RJ. Safety of using the adult omni resistance exercise scale to determine 1-rm in older men and women. Percept Mot Skills. 2011;113(2):671-6.

19. Rhea MR. Determining the magnitude of treatment effects in strength training research through the use of the effect size. J Strength Cond Res. 2004;18(4):918-20.

20. Cardozo D, Alves HB, Figueiredo T, Dias MR, Simão R. Hypotensive effect in resistance training: influence of muscle mass. ConScientiae Saúde. 2014;13(4):524-32.

21. Dias I, Salles BF, Novaes J, Costa PB, Simão R. Influence of exercise order on maximum strength in untrained young men. J Sci Med Sport. 2008;13(1):65-9.

22. Miranda H, Simão R, Vigario PDS, Salles BF, Pacheco MTT, Willardson JM. Exercise order interacts with rest interval during upper-body resistance exercise. J Strength Cond Res. 2010;24(6):1573-7.

23. 23. Spineti J, Salles BF, Rhea MR, et al. Influence of exercise order on maximum strength and muscle volume in non linear periodized resistance training. J Strength Cond Res. 2010;24(11):2962-9.

24. Simão R, Salles BF, Figueiredo T, Dias I, Willardson JM. Exercise order in resistance training. Sports Med. 2012;42(3):251-65.

25. Lizardo JHF, Simões HG. Efeitos de diferentes sessóes de exercícios resistidos sobre a hipotensão pós-exercício. Rev Bras Fisioter. 2005;9(3):289-95.

26. MacDonald JR, MacDougall JD, Hogben CD. The effects of exercising muscle mass on post exercise hypotension. Journal Hum Hypertens. 2000;14(5):317-20.

27. Mitchell JH, Payne FC, Saltin B, Schibye B. The role of muscle mass in the cardiovascular response to static contractions. J Physiol. 1980;309:45-54.

28. Seals DR, Washburn RA, Hanson PG, Painter PL, Nagle FJ. Increased cardiovascular response to static contraction of larger muscle groups. J Appl Physiol Respir Environ Exerc Physion. 1983;54(2):434-7.

29. Kenney MJ, Seals DR. Postexercise hypotension. Key features, mechanisms, and clinical significance. Hypertension. 1993;22(5):653-64.

30. Queiroz ACC, Sousa JCS, Cavalli AAP, et al. Post-resistance exercise hemodynamic and autonomic responses: Comparison between normotensive and hypertensive men. Scand J Med Sci Sports. 2015;25(4):486-94.

31. Fisher MM. The effect of resistance exercise on recovery blood pressure in normotensive and borderline hypertensive women. J Strength Cond Res. 2001;15(2):210-6.

32. Polito MD, Farinatti PTV. Consideraçôes sobre a medida da pressão arterial em exercícios contra-resistência. Rev Bras Med Esporte. 2003;9(1):25-33.

33. Polito MD, Farinatti PTV, Lira VA, Nobrega ACL. Blood pressure assessment during resistance exercise: comparison between auscultation and Finapres. Blood Press Monit. 2007;12(2):81-6.

34. Gomides RS, Costa LA, Souza DR, et al. Atenolol blunts blood pressure increase during dynamic resistance exercise in hypertensives. Br J Clin Pharmacol. 2010;70(5):664-73.

35. Miguel FM, Grings AL, Pereira GB, et al. Different cardiovascular responses to a resistance training session in hypertensive women receiving propanolol compared with normotensive controls. ScientificWorldJournal. 2012:913271.

\author{
ENDEREÇO \\ Diogo Cardozo \\ Rua Batista de Oliveira, 1145 - 36010-532 \\ Juiz de Fora - MG - BRASIL \\ e-mail: dcardozoef@gmail.com; \\ dcardozopersonaltrainer@gmail.com
}

Recebido para publicação: 19/01/2016

Revisão: 25/04/2016

Aceito: 13/06/2016 